\title{
Solutions for the MIMO Gaussian Wiretap Channel With a Cooperative Jammer
}

\author{
S. Ali A. Fakoorian, Student Member, IEEE, and A. Lee Swindlehurst, Fellow, IEEE
}

\begin{abstract}
We study the Gaussian MIMO wiretap channel with a transmitter, a legitimate receiver, an eavesdropper and an external helper, each equipped with multiple antennas. The transmitter sends confidential messages to its intended receiver, while the helper transmits jamming signals independent of the source message to confuse the eavesdropper. The jamming signal is assumed to be treated as noise at both the intended receiver and the eavesdropper. We obtain a closed-form expression for the structure of the artificial noise covariance matrix that guarantees a secrecy rate larger or at least equal to the secrecy capacity of the wiretap channel with no jamming signal. We also describe how to find specific realizations of this covariance matrix expression that provide good secrecy rate performance, even when there is no nontrivial null space between the helper and the intended receiver. Unlike prior work, our approach considers the general MIMO case, and is not restricted to SISO or MISO scenarios.
\end{abstract}

Index Terms-Cooperative jamming, interference channel, MIMO wiretap channel, physical-layer security.

\section{INTRODUCTION}

$\mathbf{R}$ ECENT information-theoretic research on secure communication has focused on enhancing security at the physical layer. The wiretap channel, first introduced and studied by Wyner [1], is the most basic physical layer model that captures the problem of communication security. This work led to the development of the notion of perfect secrecy capacity, which quantifies the maximum rate at which a transmitter can reliably send a secret message to its intended recipient, without it being decoded by an eavesdropper. The Gaussian wiretap channel, in which the outputs of the legitimate receiver and the eavesdropper are corrupted by additive white Gaussian noise, was studied in [2]. The secrecy capacity of a Gaussian wiretap channel, which is in general a difficult nonconvex optimization problem, has been addressed and solved for in [3]-[9]. The secrecy capacity under an average power constraint is treated in [4] and [5], where in [4] a beamforming approach, based on the generalized singular value decomposition (GSVD), is proposed that achieves the secrecy capacity in the high SNR regime. In [5], the necessary conditions for the optimal input

Manuscript received December 06, 2010; revised April 14, 2011; accepted June 20, 2011. Date of publication July 05, 2011; date of current version September 14, 2011. The associate editor coordinating the review of this manuscript and approving it for publication was Prof. Wolfgang Utschick. This work was supported by the U.S. Army Research Office under the Multi-University Research Initiative (MURI) grant W911NF-07-1-0318.

The authors are with the Department of Electrical Engineering and Computer Science, University of California, Irvine, CA 92697 USA (e-mail: afakoori@uci.edu; swindle@uci.edu).

Color versions of one or more of the figures in this paper are available online at http://ieeexplore.ieee.org.

Digital Object Identifier 10.1109/TSP.2011.2161298 covariance matrix are derived and it is shown that these necessary conditions can result in a closed form solution for some specific scenarios. In [7], a closed-form expression for the secrecy capacity is derived under a certain power-covariance constraint. The work in [7] is extended in [8] to the general, possibly imperfect secrecy setting by obtaining a matrix characterization of the capacity equivocation region of the MIMO Gaussian wiretap channel. Secure broadcasting with more than two receivers is considered, for instance, in [9] (and references therein) where the secrecy capacity region for the case of two legitimate receivers in the presence of an eavesdropper is characterized.

It was shown in [10] that, for a wiretap channel without feedback, a nonzero secrecy capacity can only be obtained if the eavesdropper's channel is of lower quality than that of the intended recipient. Otherwise, it is infeasible to establish a secure link under Wyner's wiretap channel model. In such situations, one approach is to exploit user cooperation in facilitating the transmission of confidential messages from the source to the destination. In [11]-[15], for example, a four-terminal relayeavesdropper channel is considered, where a source wishes to send messages to a destination while leveraging the help of a relay/helper node to hide the messages from the eavesdropper. While the relay can assist in the transmission of confidential messages, its computational cost may be prohibitive and there are difficulties associated with the coding and decoding schemes at both the relay and the intended receiver. Alternatively, a cooperating node can be used as a helper that simply transmits jamming signals, independent of the source message, to confuse the eavesdropper and increase the range of channel conditions under which secure communications can take place. The strategy of using a helper to improve the secrecy of the source-destination communication is generally known as cooperative jamming [11], [13] or noise-forwarding [14] in prior work.

In [11], the scenario where multiple single-antenna users communicate with a common receiver (i.e., the multiple access channel) in the presence of an eavesdropper is considered, and the optimal transmit power allocation that achieves the maximum secrecy sum-rate is obtained. The work of [11] shows that any user prevented from transmitting based on the obtained power allocation can help increase the secrecy rate for other users by transmitting artificial noise to the eavesdropper (cooperative jamming). In [13], a source-destination system in the presence of multiple helpers and multiple eavesdroppers is considered, where the helpers can transmit weighted jamming signals to degrade the eavesdropper's ability to decode the source. While the objective is to select the weights so as to maximize the secrecy rate under a total power constraint, or to minimize the total power under a secrecy rate constraint, the results in [13] yield suboptimal weights for both single and 
multiple eavesdroppers, due to the assumption that the jamming signal must be nulled at the destination. The noise forwarding scheme of [14] requires that the interferer's codewords be decoded by the intended receiver. A generalization of [11], [13] and [14] is proposed in [15], in which the helper's codewords do not have to be decoded by the receiver.

The prior work in [11]-[15] assumes single antenna nodes and models single-input, single-output (SISO) or multiple-input, single-output (MISO) cases. A more general MIMO case with multiple cooperative jammers was studied in [16], in which the jammers aligned their interference to lie within a pre-specified "jamming subspace" at the receiver, but the dimensions of the subspace and the power allocation were not optimized. In this paper, we also address the general MIMO case, where the transmitter, legitimate receiver, eavesdropper and helper are in general all equipped with multiple antennas. The transmitter sends confidential messages to its intended receiver, while the helper node assists the transmitter by sending jamming signals independent of the source message to confuse the eavesdropper. While the previous work on this problem shows the fundamental role of jamming as a means to increase secrecy rates, it also emphasizes the fact that noncarefully designed jamming strategies can preclude secure communication [17].

In this paper, we derive a closed-form expression for the structure of the artificial noise covariance matrix of a cooperating jammer that guarantees an achievable secrecy rate no smaller than the secrecy capacity of the wiretap channel with no jamming signal, assuming the jamming signal from the helper is treated as noise at both the intended receiver and the eavesdropper. We describe algorithms for finding specific realizations of this covariance expression that provide good secrecy rate performance, and show that even when there is no nontrivial nullspace between the helper and the intended receiver, the helper can still transmit artificial noise that does not impact the mutual information between the transmitter and the intended receiver, while decreasing the mutual information between the transmitter and the eavesdropper. Hence, the secrecy level of the confidential message is increased. The situation we consider is different from the one in [18], where the transmitter itself rather than an external helper broadcasts artificial noise to degrade the eavesdropper's channel. However, both approaches are able to achieve a positive perfect secrecy rate in scenarios where the secrecy rate in the absence of jamming is zero.

The remainder of the paper is organized as follows. In Section II, we describe the system model for the helper-assisted Gaussian MIMO wiretap channel and formulate the problem to be solved. In Sections III and IV, we derive the artificial noise covariance matrix that guarantees an achievable secrecy rate no smaller than the secrecy capacity of the wiretap channel with no jamming signal, and we derive a lower bound on the improvement in secrecy rate that results from addition of the cooperative jammer. Numerical results in Section $\mathrm{V}$ are presented to illustrate the proposed solution. Finally, Section VI concludes the paper.

Notation: Throughout the paper, we use boldface uppercase letters to denote matrices. Vector-valued random variables are written with nonboldface uppercase letters (e.g., $X$ ), while the corresponding lowercase boldface letter $(\mathbf{x})$ denotes a specific realization of the random variable. Scalar variables are written

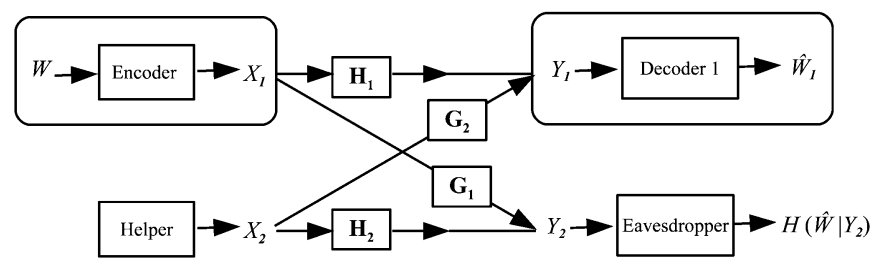

Fig. 1. System model for the Gaussian MIMO wiretap channel with an external helper.

with nonboldface (lowercase or uppercase) letters. We use $(\cdot)^{T}$ to represent matrix transposition, $(\cdot)^{H}$ the Hermitian (i.e., conjugate) transpose, $\operatorname{Tr}(\cdot)$ the matrix trace, $E$ the expectation operator, $\mathbf{I}$ the identity matrix, and $\mathbf{0}$ a matrix or vector with all zeros. Mutual information between the random variables $A$ and $B$ is denoted by $I(A ; B)$, and $\mathcal{C N}(0,1)$ represents the complex circularly symmetric Gaussian distribution with zero mean and unit variance.

\section{SYSTEM MODEL}

We consider a MIMO wiretap channel that includes a transmitter, an intended receiver, a cooperative jammer and an eavesdropper, with $n_{t}, n_{r}, n_{h}$ and $n_{e}$ antennas, respectively. The transmitter sends a confidential message to the intended receiver with the aid of the helper, in the presence of an eavesdropper. We assume that the helper does not know the confidential message and transmits only a Gaussian jamming signal which is not known at the intended receiver nor the eavesdropper and which is treated as noise at both receivers. The mathematical model for this scenario, shown in Fig. 1, is given by

$$
\begin{aligned}
& \mathbf{y}_{1}=\mathbf{H}_{1} \mathbf{x}_{1}+\mathbf{G}_{2} \mathbf{x}_{2}+\mathbf{z}_{1} \\
& \mathbf{y}_{2}=\mathbf{H}_{2} \mathbf{x}_{2}+\mathbf{G}_{1} \mathbf{x}_{1}+\mathbf{z}_{2}
\end{aligned}
$$

where $\mathbf{x}_{1}$ is a zero-mean $n_{t} \times 1$ transmitted signal vector, $\mathbf{x}_{2}$ is a zero-mean $n_{h} \times 1$ jamming vector transmitted by the helper, and $\mathbf{z}_{1} \in \mathbb{C}^{n_{r} \times 1}, \mathbf{z}_{2} \in \mathbb{C}^{n_{e} \times 1}$ are additive white Gaussian noise (AWGN) vectors at the intended receiver and the eavesdropper, respectively, with i.i.d. entries distributed as $\mathcal{C N}(0,1)$. The matrices $\mathbf{H}_{1}, \mathbf{G}_{1}$ represent the channels from the transmitter to the intended receiver and eavesdropper, respectively, while $\mathbf{H}_{2}, \mathbf{G}_{2}$ are the channels from the helper to the eavesdropper and intended receiver, respectively. The channels are assumed to be independent of each other and full rank with arbitrary dimensions. We also assume that full channel state information is available to all parties, including the eavesdropper. This is possible in situations where the eavesdropper is normally an active member of the network, transmitting and receiving nonconfidential information from the other parties in other time slots.

The jamming signal transmitted by the helper satisfies an average power constraint:

$$
\operatorname{Tr}\left(E\left\{X_{2} X_{2}^{H}\right\}\right)=\operatorname{Tr}\left(\mathbf{K}_{w}\right) \leq P_{h}
$$

where $X_{2}$ is the random variable associated with the specific realization $\mathbf{x}_{2}$ and $\mathbf{K}_{w}$ is the corresponding covariance matrix. The channel input is subject to a matrix power constraint [7] [19]

$$
E\left\{X_{1} X_{1}^{H}\right\}=\mathbf{K}_{x} \preceq \mathbf{S}
$$

where $\mathbf{K}_{x}$ is the input covariance matrix, $\mathbf{S}$ is a positive semidefinite matrix, and "”" denotes that $\mathbf{S}-\mathbf{K}_{x}$ is positive semidefi- 
nite. Note that (4) is a rather general power constraint that subsumes many other important power constraints, including the average total and per-antenna power constraints as special cases. The approach developed in this paper will assume that $P_{h}$ and $\mathbf{S}$ (or $\operatorname{Tr}(\mathbf{S}) \leq P_{t}$ ) are fixed, and that power is not allocated jointly between the transmitter and helper. The numerical results presented later, however, will illustrate the trade-off associated with the power allocation when $P_{h}+P_{t}$ is fixed.

As mentioned before, we assume Gaussian signaling for the helper. Thus the effective noise at both receivers is Gaussian and consequently the above MIMO wiretap channel model is Gaussian. For this case, a Gaussian input signal is the optimal choice [6], [19]. Hence, the general optimization problem is equivalent to finding the matrices $\mathbf{K}_{x} \succeq 0$ and $\mathbf{K}_{w} \succeq 0$ that allow the secrecy capacity of the network to be obtained. A matrix characterization of this optimization problem is given by:

$$
\begin{aligned}
C_{\mathrm{sec}}= & \max _{\mathbf{K}_{x} \succeq 0, \mathbf{K}_{w} \succeq 0}\left[I\left(X_{1} ; Y_{1}\right)-I\left(X_{1} ; Y_{2}\right)\right] \\
= & \max _{\mathbf{K}_{x} \succeq 0, \mathbf{K}_{w} \succeq 0} \log \left|\mathbf{K}_{x} \mathbf{H}_{1}^{H}\left(\mathbf{G}_{2} \mathbf{K}_{w} \mathbf{G}_{2}^{H}+\mathbf{I}\right)^{-1} \mathbf{H}_{1}+\mathbf{I}\right| \\
& -\log \left|\mathbf{K}_{x} \mathbf{G}_{1}^{H}\left(\mathbf{H}_{2} \mathbf{K}_{w} \mathbf{H}_{2}^{H}+\mathbf{I}\right)^{-1} \mathbf{G}_{1}+\mathbf{I}\right|
\end{aligned}
$$

where the nonconvex maximization problem is carried out under the power constraints given in (3) and (4).

Lemma 1: For a given $\mathbf{K}_{w}$, the maximum achievable secrecy rate is given by

$$
R_{\mathrm{sec}}(\mathbf{S})=\sum_{i=1}^{\rho} \log \gamma_{i}
$$

where $\gamma_{i}, i=1, \ldots, \rho$, are the generalized eigenvalues of the pencil

$$
\begin{aligned}
&\left(\mathbf{S}^{\frac{1}{2}} \mathbf{H}_{1}^{H}\left(\mathbf{G}_{2} \mathbf{K}_{w} \mathbf{G}_{2}^{H}+\mathbf{I}\right)^{-1} \mathbf{H}_{1} \mathbf{S}^{\frac{1}{2}}+\mathbf{I},\right. \\
&\left.\mathbf{S}^{\frac{1}{2}} \mathbf{G}_{1}^{H}\left(\mathbf{H}_{2} \mathbf{K}_{w} \mathbf{H}_{2}^{H}+\mathbf{I}\right)^{-1} \mathbf{G}_{1} \mathbf{S}^{\frac{1}{2}}+\mathbf{I}\right)
\end{aligned}
$$

that are greater than 1.

Proof: For a given $\mathbf{K}_{w}$, the optimization problem in (5) is performed only over $\mathbf{K}_{x}$ under the matrix power constraint (4). Thus it is equivalent to a simple MIMO Gaussian wiretap channel without a helper, where the noise covariance matrices at the receiver and the eavesdropper are $\left(\mathbf{G}_{2} \mathbf{K}_{w} \mathbf{G}_{2}^{H}+\mathbf{I}\right)$ and $\left(\mathbf{H}_{2} \mathbf{K}_{w} \mathbf{H}_{2}^{H}+\mathbf{I}\right)$, respectively. In other words, for a given $\mathbf{K}_{w}$ employed by the helper, the maximum achievable secrecy rate for the wiretap channel with helper is the same as the secrecy capacity of the equivalent wiretap channel without a helper, but with spatially colored noise. This lemma is a simple extension of [7] and ([19, Theorem 3]) for the standard MIMO Gaussian wiretap channel without a helper.

Note that since both elements of the pencil (7) are strictly positive definite, all of the generalized eigenvalues are real and positive [19], [20]. In (6), a total of $\rho$ of them are assumed to be greater than one. Clearly, if there are no such eigenvalues, then the information signal received at the intended receiver is a degraded version of that of the eavesdropper, and in this case the secrecy rate for such a $\mathbf{K}_{w}$ is zero. Note also that Lemma 1 only provides the maximum secrecy rate for the optimal $\mathbf{K}_{x}$, but does not give an explicit expression for this $\mathbf{K}_{x}$. A general expression for the maximizing $\mathbf{K}_{x}$ will be given in the next section. Finally note that the secrecy rate given in (6) might in general be less than the secrecy capacity of the wiretap channel with no jamming signal $\left(\mathbf{K}_{w}=0\right)$ if $\mathbf{K}_{w}$ is not properly designed.

To solve the general optimization problem in (5), we would need to find the $\mathbf{K}_{w}$ that maximizes (6). Unfortunately, this appears to be a very difficult problem to solve without resorting to some type of ad hoc search. In the following we obtain a suboptimal closed-form solution for the artificial noise covariance matrix $\mathbf{K}_{w}$ that guarantees no decrease in the mutual information between the transmitter and the intended receiver compared with the case where $\mathbf{K}_{w}=\mathbf{0}$, while maintaining the power constraint in (3). Hence, the new nonzero $\mathbf{K}_{w}$ will only interfere with the eavesdropper, and the secrecy level of the confidential message will be increased. Once such a $\mathbf{K}_{w}$ is found, additional improvement in the secrecy rate can be achieved if the transmitter updates its covariance matrix $\mathbf{K}_{x}$ for the obtained $\mathbf{K}_{w}$. The final secrecy rate for this method is obtained by simply computing (6) and (7) for the resulting $\mathbf{K}_{w}$. Note that we will not propose an iterative algorithm that would further alternate between calculating $\mathbf{K}_{x}$ and $\mathbf{K}_{w}$. We will see in the next section that there is no obvious way to update $\mathbf{K}_{w}$ from a known nonzero value.

\section{ANALyTiCAL MethoD}

\section{A. Solution Without a Helper}

We begin with the case where the helper transmits no signal $\left(\mathbf{K}_{w}=0\right)$. In this case, the communication system is reduced to a simple MIMO Gaussian wiretap channel without helper. Based on Lemma 1, the maximum of (5) when $\mathbf{K}_{w}=0$ is obtained by applying the generalized eigenvalue decomposition to the following two Hermitian positive definite matrices [7], [19]:

$$
\mathbf{S}^{\frac{1}{2}} \mathbf{H}_{1}^{H} \mathbf{H}_{1} \mathbf{S}^{\frac{1}{2}}+\mathbf{I}, \quad \mathbf{S}^{\frac{1}{2}} \mathbf{G}_{1}^{H} \mathbf{G}_{1} \mathbf{S}^{\frac{1}{2}}+\mathbf{I} .
$$

In particular, there exists an invertible generalized eigenvector matrix $\mathbf{C}$ such that [20]

$$
\begin{aligned}
& \mathbf{C}^{H}\left[\mathbf{S}^{\frac{1}{2}} \mathbf{G}_{1}^{H} \mathbf{G}_{1} \mathbf{S}^{\frac{1}{2}}+\mathbf{I}\right] \mathbf{C}=\mathbf{I} \\
& \mathbf{C}^{H}\left[\mathbf{S}^{\frac{1}{2}} \mathbf{H}_{1}^{H} \mathbf{H}_{1} \mathbf{S}^{\frac{1}{2}}+\mathbf{I}\right] \mathbf{C}=\mathbf{\Lambda}
\end{aligned}
$$

where $\boldsymbol{\Lambda}=\operatorname{diag}\left\{\lambda_{1}, \ldots, \lambda_{n_{t}}\right\}$ is a positive definite diagonal matrix and $\lambda_{1}, \ldots, \lambda_{n_{t}}$ represent the generalized eigenvalues. Without loss of generality, we assume the generalized eigenvalues are ordered as

$$
\lambda_{1} \geq \cdots \geq \lambda_{b}>1 \geq \lambda_{b+1} \geq \cdots \geq \lambda_{n_{t}}>0
$$

so that a total of $b\left(0 \leq b \leq n_{t}\right)$ are assumed to be greater than 1 . Hence, we can write $\Lambda$ as

$$
\boldsymbol{\Lambda}=\left[\begin{array}{cc}
\boldsymbol{\Lambda}_{1} & 0 \\
0 & \boldsymbol{\Lambda}_{2}
\end{array}\right]
$$

where $\boldsymbol{\Lambda}_{1}=\operatorname{diag}\left\{\lambda_{1}, \ldots, \lambda_{b}\right\}$ and $\boldsymbol{\Lambda}_{2}=$ $\operatorname{diag}\left\{\lambda_{b+1}, \ldots, \lambda_{n_{t}}\right\}$. Also, we can write $\mathbf{C}$ as

$$
\mathbf{C}=\left[\begin{array}{ll}
\mathbf{C}_{1} & \mathbf{C}_{2}
\end{array}\right]
$$

where $\mathbf{C}_{1}$ is the $n_{t} \times b$ submatrix representing the generalized eigenvectors corresponding to $\left\{\lambda_{1}, \ldots, \lambda_{b}\right\}$ and $\mathbf{C}_{2}$ is the $n_{t} \times$ 
$\left(n_{t}-b\right)$ submatrix representing the generalized eigenvectors corresponding to $\left\{\lambda_{b+1}, \ldots, \lambda_{n_{t}}\right\}$.

For the case of $\mathbf{K}_{w}=0$, the maximum achievable secrecy rate is the secrecy capacity of the standard wiretap channel with no jamming signal, under the matrix power constraint in (4), and is given by (Lemma 1 or [19, Th. 3]

$$
C_{s}=\sum_{i=1}^{b} \log \lambda_{i}=\log \left|\boldsymbol{\Lambda}_{1}\right|
$$

where the input covariance matrix $\mathbf{K}_{x}^{*}$ that maximizes (5) is given by [7], [19]

$$
\mathbf{K}_{x}^{*}=\mathbf{S}^{\frac{1}{2}} \mathbf{C}\left[\begin{array}{cc}
\left(\mathbf{C}_{1}^{H} \mathbf{C}_{1}\right)^{-1} & 0 \\
0 & 0
\end{array}\right] \mathbf{C}^{H} \mathbf{S}^{\frac{1}{2}} .
$$

Note that (13) is a general expression for the $\mathbf{K}_{x}$ that optimizes (5) for a given $\mathbf{K}_{w}$ even when $\mathbf{K}_{w} \neq 0$, although in this case the $\mathbf{C}$ will be the generalized eigenvector matrix of the pencil in (7). From (9) we note that $\mathbf{H}_{1}^{H} \mathbf{H}_{1}$ can be written as

$$
\mathbf{H}_{1}^{H} \mathbf{H}_{1}=\mathbf{S}^{-1 / 2}\left[\mathbf{C}^{-H}\left[\begin{array}{cc}
\boldsymbol{\Lambda}_{1} & 0 \\
0 & \boldsymbol{\Lambda}_{2}
\end{array}\right] \mathbf{C}^{-1}-\mathbf{I}\right] \mathbf{S}^{-1 / 2} .
$$

The following lemma gives the mutual information $I\left(X_{1} ; Y_{1}\right)$ between the transmitter and the intended receiver when $\mathbf{K}_{w}=0$ and $\mathbf{K}_{x}$ is given by (13).

Lemma 2: The following equality holds:

$$
\begin{aligned}
\left.I\left(X_{1} ; Y_{1}\right)\right|_{\mathbf{K}_{w}=0, \mathbf{K}_{x}=\mathbf{K}_{x}^{*}} & =\log \left|\mathbf{K}_{x}^{*} \mathbf{H}_{1}^{H} \mathbf{H}_{1}+\mathbf{I}\right| \\
& =\log \left|\left(\mathbf{C}_{1}^{H} \mathbf{C}_{1}\right)^{-1} \boldsymbol{\Lambda}_{1}\right| .
\end{aligned}
$$

Proof: Following the same steps as the proof of ([7, App. D]) and using (13) and (14), we have

$$
\begin{aligned}
& \left|\mathbf{K}_{x}^{*} \mathbf{H}_{1}^{H} \mathbf{H}_{1}+\mathbf{I}\right| \\
& =\mid \mathbf{S}^{\frac{1}{2}} \mathbf{C}\left[\begin{array}{cc}
\left(\mathbf{C}_{1}^{H} \mathbf{C}_{1}\right)^{-1} & 0 \\
0 & 0
\end{array}\right] \mathbf{C}^{H} \\
& \times\left[\mathbf{C}^{-H}\left[\begin{array}{cc}
\boldsymbol{\Lambda}_{1} & 0 \\
0 & \boldsymbol{\Lambda}_{2}
\end{array}\right] \mathbf{C}^{-1}-\mathbf{I}\right] \mathbf{S}^{-1 / 2}+\mathbf{I} \mid \\
& =\mid\left[\begin{array}{cc}
\left(\mathbf{C}_{1}^{H} \mathbf{C}_{1}\right)^{-1} & 0 \\
0 & 0
\end{array}\right] \times\left[\begin{array}{cc}
\boldsymbol{\Lambda}_{1} & 0 \\
0 & \boldsymbol{\Lambda}_{2}
\end{array}\right] \\
& \text { - }\left[\begin{array}{cc}
\left(\mathbf{C}_{1}^{H} \mathbf{C}_{1}\right)^{-1} & 0 \\
0 & 0
\end{array}\right] \mathbf{C}^{H} \mathbf{C}+\mathbf{I} \mid \\
& =\mid\left[\begin{array}{cc}
\left(\mathbf{C}_{1}^{H} \mathbf{C}_{1}\right)^{-1} \boldsymbol{\Lambda}_{1} & 0 \\
0 & 0
\end{array}\right] \\
& -\left[\begin{array}{cc}
\mathbf{I} & \left(\mathbf{C}_{1}^{H} \mathbf{C}_{1}\right)^{-1} \mathbf{C}_{1}^{H} \mathbf{C}_{2} \\
0 & 0
\end{array}\right]+\mathbf{I} \mid \\
& =\left|\left[\begin{array}{cc}
\left(\mathbf{C}_{1}^{H} \mathbf{C}_{1}\right)^{-1} \boldsymbol{\Lambda}_{1} & -\left(\mathbf{C}_{1}^{H} \mathbf{C}_{1}\right)^{-1} \mathbf{C}_{1}^{H} \mathbf{C}_{2} \\
0 & \mathbf{I}
\end{array}\right]\right| \\
& =\left|\left(\mathbf{C}_{1}^{H} \mathbf{C}_{1}\right)^{-1} \boldsymbol{\Lambda}_{1}\right|
\end{aligned}
$$

where (16) follows from the fact that $|\mathbf{A B}+\mathbf{I}|=|\mathbf{B A}+\mathbf{I}|$, and (17) follows since

$$
\mathbf{C}^{H} \mathbf{C}=\left[\begin{array}{ll}
\mathbf{C}_{1} & \mathbf{C}_{2}
\end{array}\right]^{H}\left[\begin{array}{ll}
\mathbf{C}_{1} & \mathbf{C}_{2}
\end{array}\right]=\left[\begin{array}{ll}
\mathbf{C}_{1}^{H} \mathbf{C}_{1} & \mathbf{C}_{1}^{H} \mathbf{C}_{2} \\
\mathbf{C}_{2}^{H} \mathbf{C}_{1} & \mathbf{C}_{2}^{H} \mathbf{C}_{2}
\end{array}\right] .
$$

\section{B. Solution With a Helper}

We now return to the general optimization problem in (5) with nonzero $\mathbf{K}_{w}$. As the helper begins to broadcast artificial noise, both the mutual information between the transmitter and the intended receiver $I\left(X_{1} ; Y_{1}\right)$ and the mutual information between the transmitter and the eavesdropper $I\left(X_{1} ; Y_{2}\right)$ are in general decreased. Both of these functions are nonincreasing in $\mathbf{K}_{w}$ since

$$
\frac{|\mathbf{A}+\mathbf{B}|}{|\mathbf{B}|} \geq \frac{|\mathbf{A}+\mathbf{B}+\Delta|}{|\mathbf{B}+\Delta|}
$$

when $\mathbf{A}, \Delta \succeq 0$ and $\mathbf{B} \succ 0$ [22]. A favorable choice for $\mathbf{K}_{w}$ would be one that reduces $I\left(X_{1} ; Y_{2}\right)$ more than $I\left(X_{1} ; Y_{1}\right)$. Since the optimal solution to (5) is intractable, we propose a suboptimal approach that introduces an additional constraint; namely, we search among those $\mathbf{K}_{w}$ matrices that guarantee no decrease in the favorable term $I\left(X_{1} ; Y_{1}\right)$ while the power constraint (3) is satisfied. It should be noted that this approach is more general than the cooperative jamming schemes proposed in [12], [13] for the MISO case where the jamming signal is nulled out at the destination. Clearly, such suboptimal solutions are restricted to the case where there exists a null space between the helper and the intended receiver.

In the following, we obtain an expression that represents all $\mathbf{K}_{w} \succeq 0$ matrices with the power constraint $\operatorname{Tr}\left(\mathbf{K}_{w}\right)=P_{h}$ that do not impact the mutual information between the transmitter and the intended receiver; i.e.

$$
\left.I\left(X_{1} ; Y_{1}\right)\right|_{\mathbf{K}_{w} \succeq 0, \mathbf{K}_{x}=\mathbf{K}_{x}^{*}}=\left.I\left(X_{1} ; Y_{1}\right)\right|_{\mathbf{K}_{w}=0, \mathbf{K}_{x}=\mathbf{K}_{x}^{*}}
$$

or from (15)

$$
\begin{aligned}
& \log \left|\mathbf{K}_{x}^{*} \mathbf{H}_{1}^{H}\left(\mathbf{G}_{2} \mathbf{K}_{w} \mathbf{G}_{2}^{H}+\mathbf{I}\right)^{-1} \mathbf{H}_{1}+\mathbf{I}\right| \\
& \quad=\log \left|\mathbf{K}_{x}^{*} \mathbf{H}_{1}^{H} \mathbf{H}_{1}+\mathbf{I}\right| \\
& \quad=\log \left|\left(\mathbf{C}_{1}^{H} \mathbf{C}_{1}\right)^{-1} \boldsymbol{\Lambda}_{1}\right| .
\end{aligned}
$$

Note that, without loss of generality, we have used an equality power constraint $\operatorname{Tr}\left(\mathbf{K}_{w}\right)=P_{h}$ since for the desired $\mathbf{K}_{w}$ the best performance is in general obtained when helper transmits at maximum power.

Theorem 1: All $\mathbf{K}_{w} \succeq 0$ matrices for which

$$
\begin{aligned}
& \log \left|\mathbf{K}_{x}^{*} \mathbf{H}_{1}^{H}\left(\mathbf{G}_{2} \mathbf{K}_{w} \mathbf{G}_{2}^{H}+\mathbf{I}\right)^{-1} \mathbf{H}_{1}+\mathbf{I}\right| \\
& \quad=\log \left|\mathbf{K}_{x}^{*} \mathbf{H}_{1}^{H} \mathbf{H}_{1}+\mathbf{I}\right| \\
& \quad=\log \left|\left(\mathbf{C}_{1}^{H} \mathbf{C}_{1}\right)^{-1} \boldsymbol{\Lambda}_{1}\right|
\end{aligned}
$$

satisfy the following relation:

$$
\begin{aligned}
\mathbf{H}_{1}^{H}\left(\mathbf{G}_{2} \mathbf{K}_{w} \mathbf{G}_{2}^{H}+\mathbf{I}\right)^{-1} \mathbf{H}_{1} \\
\quad=\mathbf{S}^{-1 / 2}\left[\mathbf{C}^{-H}\left[\begin{array}{cc}
\boldsymbol{\Lambda}_{1} & 0 \\
0 & \mathbf{N}
\end{array}\right] \mathbf{C}^{-1}-\mathbf{I}\right] \mathbf{S}^{-1 / 2}
\end{aligned}
$$

where

$$
\begin{aligned}
& \boldsymbol{\Lambda}_{22} \preceq \mathbf{N} \preceq \boldsymbol{\Lambda}_{2} \\
& \boldsymbol{\Lambda}_{22}=\mathbf{C}_{2}^{H} \mathbf{C}_{2}+\mathbf{C}_{2}^{H} \mathbf{C}_{1}\left(\boldsymbol{\Lambda}_{1}-\mathbf{C}_{1}^{H} \mathbf{C}_{1}\right)^{-1} \mathbf{C}_{1}^{H} \mathbf{C}_{2}
\end{aligned}
$$


Proof: In Appendix A, using similar steps as those used to obtain (18), we show that all $\boldsymbol{\Sigma} \succ 0$ matrices for which $\log \left|\mathbf{K}_{x}^{*} \boldsymbol{\Sigma}+\mathbf{I}\right|=\log \left|\left(\mathbf{C}_{1}^{H} \mathbf{C}_{1}\right)^{-1} \boldsymbol{\Lambda}_{1}\right|$ must have the following form:

$$
\boldsymbol{\Sigma}=\mathbf{S}^{-1 / 2}\left[\mathbf{C}^{-H}\left[\begin{array}{cc}
\boldsymbol{\Lambda}_{1} & \mathbf{M} \\
\mathbf{M}^{H} & \mathbf{N}
\end{array}\right] \mathbf{C}^{-1}-\mathbf{I}\right] \mathbf{S}^{-1 / 2}
$$

In the following, we obtain matrices $\mathbf{N} \succeq 0$ and $\mathbf{M}$ and complete the proof by considering the following specific choice for $\Sigma$ :

$$
\boldsymbol{\Sigma}=\mathbf{H}_{1}^{H}\left(\mathbf{G}_{2} \mathbf{K}_{w} \mathbf{G}_{2}^{H}+\mathbf{I}\right)^{-1} \mathbf{H}_{1} .
$$

For the specific $\boldsymbol{\Sigma}$ in (23), it is evident that

$$
0 \preceq \boldsymbol{\Sigma} \preceq \mathbf{H}_{1}^{H} \mathbf{H}_{1} \text {. }
$$

By applying the constraint $\boldsymbol{\Sigma} \preceq \mathbf{H}_{1}^{H} \mathbf{H}_{1}$ on (22) and using (14), it is enough to show that

$$
\left[\begin{array}{cc}
\boldsymbol{\Lambda}_{1} & \mathbf{M} \\
\mathbf{M}^{H} & \mathbf{N}
\end{array}\right] \preceq\left[\begin{array}{cc}
\boldsymbol{\Lambda}_{1} & 0 \\
0 & \boldsymbol{\Lambda}_{2}
\end{array}\right]
$$

or equivalently that

$$
\left[\begin{array}{cc}
0 & -\mathbf{M} \\
-\mathbf{M}^{H} & \mathbf{\Lambda}_{2}-\mathbf{N}
\end{array}\right] \succeq 0 .
$$

By applying the Schur Complement Lemma [20], the above relationship is true iff $\boldsymbol{\Lambda}_{2}-\mathbf{N} \succeq 0$ and $-\mathbf{M}\left(\boldsymbol{\Lambda}_{2}-\mathbf{N}\right)^{-1} \mathbf{M}^{H} \succeq 0$, which in turn is true only when

$$
\begin{aligned}
\mathbf{M} & =0 \\
\boldsymbol{\Lambda}_{2}-\mathbf{N} & \succeq \quad 0 .
\end{aligned}
$$

Applying the results of (25) and (26) in (22) for the specific choice of $\boldsymbol{\Sigma}=\mathbf{H}_{1}^{H}\left(\mathbf{G}_{2} \mathbf{K}_{w} \mathbf{G}_{2}^{H}+\mathbf{I}\right)^{-1} \mathbf{H}_{1}$, we have

$$
\boldsymbol{\Sigma}=\mathbf{S}^{-1 / 2}\left[\mathbf{C}^{-H}\left[\begin{array}{cc}
\boldsymbol{\Lambda}_{1} & 0 \\
0 & \mathbf{N}
\end{array}\right] \mathbf{C}^{-1}-\mathbf{I}\right] \mathbf{S}^{-1 / 2} .
$$

Based on (24), we also need to show that $\Sigma \succeq 0$. From (27), it is enough to show that

$$
\left[\begin{array}{cc}
\boldsymbol{\Lambda}_{1} & 0 \\
0 & \mathbf{N}
\end{array}\right]-\mathbf{C}^{H} \mathbf{C}=\left[\begin{array}{cc}
\boldsymbol{\Lambda}_{1}-\mathbf{C}_{1}^{H} \mathbf{C}_{1} & -\mathbf{C}_{1}^{H} \mathbf{C}_{2} \\
-\mathbf{C}_{2}^{H} \mathbf{C}_{1} & \mathbf{N}-\mathbf{C}_{2}^{H} \mathbf{C}_{2}
\end{array}\right] \succeq 0 .
$$

By applying the Schur Complement Lemma, the above relationship is true iff $\boldsymbol{\Lambda}_{1}-\mathbf{C}_{1}^{H} \mathbf{C}_{1} \succeq 0$ and $\mathbf{N}-\mathbf{C}_{2}^{H} \mathbf{C}_{2}-\mathbf{C}_{2}^{H} \mathbf{C}_{1}\left(\boldsymbol{\Lambda}_{1}-\right.$ $\left.\mathbf{C}_{1}^{H} \mathbf{C}_{1}\right)^{-1} \mathbf{C}_{1}^{H} \mathbf{C}_{2} \succeq 0$. Using (8)-(10), it is evident that

$$
\begin{aligned}
\boldsymbol{\Lambda}_{1}-\mathbf{C}_{1}^{H} \mathbf{C}_{1} & =\mathbf{C}_{1}^{H}\left[\mathbf{S}^{\frac{1}{2}} \mathbf{H}_{1}^{H} \mathbf{H}_{1} \mathbf{S}^{\frac{1}{2}}+\mathbf{I}\right] \mathbf{C}_{1}-\mathbf{C}_{1}^{H} \mathbf{C}_{1} \\
& =\mathbf{C}_{1}^{H} \mathbf{S}^{\frac{1}{2}} \mathbf{H}_{1}^{H} \mathbf{H}_{1} \mathbf{S}^{\frac{1}{2}} \mathbf{C}_{1} \succeq 0
\end{aligned}
$$

and finally the lower bound for $\mathbf{N}$ is given by $\mathbf{N} \succeq$ $\mathbf{C}_{2}^{H} \mathbf{C}_{2}+\mathbf{C}_{2}^{H} \mathbf{C}_{1}\left(\boldsymbol{\Lambda}_{1}-\mathbf{C}_{1}^{H} \mathbf{C}_{1}\right)^{-1} \mathbf{C}_{1}^{H} \mathbf{C}_{2} \succ 0$, which completes the proof.

Note that as $\mathbf{N} \rightarrow \boldsymbol{\Lambda}_{22}$, we have $\operatorname{Tr}\left(\mathbf{K}_{w}\right) \rightarrow \infty$. Moreover, $\operatorname{Tr}\left(\mathbf{K}_{w}\right)=0$ is achieved by $\mathbf{N}=\boldsymbol{\Lambda}_{2}$. Hence, for each scalar $P_{h}$, there always exists an $\mathbf{N}$ in the range $\boldsymbol{\Lambda}_{22} \preceq \mathbf{N} \preceq \boldsymbol{\Lambda}_{2}$ that will lead to a $\mathbf{K}_{w}$ that satisfies (20) with $\operatorname{Tr}\left(\mathbf{K}_{w}\right)=P_{h}$.

So far, we have obtained an expression for the covariance matrix of the artificial noise transmitted by the helper that guarantees no decrease in the secrecy rate of the channel compared with the case of no jamming signal. A key question however is how much the secrecy rate has been increased, due to the effect of the artificial noise in reducing the mutual information available to the eavesdropper. While this can be evaluated numerically (and in the simulations presented later we will see that the addition of the helper can dramatically improve the secrecy rate), it is difficult to quantify the improvement analytically, especially for the general MIMO scenario. Instead, in the discussion later, we provide an approximate lower bound on the minimum improvement in the secrecy rate that is achievable using the above results.

\section{A Lower Bound on the Secrecy Rate Improvement}

The lower bound derived in this section assumes the helper is using a covariance matrix $\mathbf{K}_{w}$ that satisfies (20), and that the transmitter's covariance matrix $\mathbf{K}_{x}$ has not been updated from the optimal value in (13) obtained when $\mathbf{K}_{w}=\mathbf{0}$. Obviously, additional improvement in the secrecy rate will in general be obtained once $\mathbf{K}_{x}$ is updated to reflect the presence of the helper's jamming signal.

Let $C_{s}$ represent the secrecy capacity of the wiretap channel without helper $\left(\mathbf{K}_{w}=\mathbf{0}\right)$ and let $R_{s H}$ represent the secrecy rate of the MIMO wiretap channel with helper when the transmitter covariance matrix is given by (13) and the helper's covariance matrix satisfies (20). We have

$$
\begin{aligned}
R_{s H} & -C_{s} \\
= & \left.\left(I\left(X_{1} ; Y_{1}\right)-I\left(X_{1} ; Y_{2}\right)\right)\right|_{\mathbf{K}_{w} \succeq 0, \mathbf{K}_{x}^{*}} \\
& -\left.\left(I\left(X_{1} ; Y_{1}\right)-I\left(X_{1} ; Y_{2}\right)\right)\right|_{\mathbf{K}_{w}=0, \mathbf{K}_{x}^{*}} \\
= & \left.I\left(X_{1} ; Y_{2}\right)\right|_{\mathbf{K}_{w}=0, \mathbf{K}_{x}^{*}}-\left.I\left(X_{1} ; Y_{2}\right)\right|_{\mathbf{K}_{w} \succeq 0, \mathbf{K}_{x}^{*}} \\
= & \log \left|\mathbf{K}_{x}^{*} \mathbf{G}_{1}^{H} \mathbf{G}_{1}+\mathbf{I}\right| \\
& -\log \left|\mathbf{K}_{x}^{* \frac{1}{2}} \mathbf{G}_{1}^{H}\left(\mathbf{H}_{2} \mathbf{K}_{w} \mathbf{H}_{2}^{H}+\mathbf{I}\right)^{-1} \mathbf{G}_{1} \mathbf{K}_{x}^{* \frac{1}{2}}+\mathbf{I}\right|
\end{aligned}
$$

where (28) comes from Theorem 1. Hence to obtain a lower bound on $R_{s H}-C_{s}$ we need to obtain an upper bound on $\left.I\left(X_{1} ; Y_{2}\right)\right|_{\mathbf{K}_{w} \succeq 0, \mathbf{K}_{x}^{*}}$. We have

$$
\begin{aligned}
& \log \left|\mathbf{K}_{x}^{* \frac{1}{2}} \mathbf{G}_{1}^{H}\left(\mathbf{H}_{2} \mathbf{K}_{w} \mathbf{H}_{2}^{H}+\mathbf{I}\right)^{-1} \mathbf{G}_{1} \mathbf{K}_{x}^{* \frac{1}{2}}+\mathbf{I}\right| \\
& =\log \mid \mathbf{K}_{x}^{* \frac{1}{2}} \mathbf{G}_{1}^{H} \mathbf{G}_{1} \mathbf{K}_{x}^{* \frac{1}{2}}+\mathbf{I} \\
& -\mathbf{K}_{x}^{* \frac{1}{2}} \mathbf{G}_{1}^{H} \mathbf{H}_{2} \mathbf{K}_{w}{ }^{\frac{1}{2}}\left(\mathbf{K}_{w}^{\frac{1}{2}} \mathbf{H}_{2}^{H} \mathbf{H}_{2} \mathbf{K}_{w}^{\frac{1}{2}}+\mathbf{I}\right)^{-1} \\
& \times \mathbf{K}_{w}^{\frac{1}{2}} \mathbf{H}_{2}^{H} \mathbf{G}_{1} \mathbf{K}_{x}^{* \frac{1}{2}} \\
& =\log \mid \mathbf{K}_{x}^{* \frac{1}{2}} \mathbf{G}_{1}^{H} \mathbf{G}_{1} \mathbf{K}_{x}^{* \frac{1}{2}}+2 \mathbf{I} \\
& -\mathbf{K}_{x}^{* \frac{1}{2}} \mathbf{G}_{1}^{H} \mathbf{H}_{2} \mathbf{K}_{w^{\frac{1}{2}}}\left(\mathbf{K}_{w^{\frac{1}{2}}} \mathbf{H}_{2}^{H} \mathbf{H}_{2} \mathbf{K}_{w}^{\frac{1}{2}}+\mathbf{I}\right)^{-1} \\
& \times \mathbf{K}_{w}^{\frac{1}{2}} \mathbf{H}_{2}^{H} \mathbf{G}_{1} \mathbf{K}_{x}^{* \frac{1}{2}}-\mathbf{I} \\
& \leq \log \left(\left|\mathbf{K}_{x}^{* \frac{1}{2}} \mathbf{G}_{1}^{H} \mathbf{G}_{1} \mathbf{K}_{x}^{* \frac{1}{2}}+2 \mathbf{I}\right|\right. \\
& -\mid \mathbf{K}_{x}^{* \frac{1}{2}} \mathbf{G}_{1}^{H} \mathbf{H}_{2} \mathbf{K}_{w}^{\frac{1}{2}}\left(\mathbf{K}_{w}^{\frac{1}{2}} \mathbf{H}_{2}^{H} \mathbf{H}_{2} \mathbf{K}_{w}^{\frac{1}{2}}+\mathbf{I}\right)^{-1} \\
& \left.\times \mathbf{K}_{w}^{\frac{1}{2}} \mathbf{H}_{2}^{H} \mathbf{G}_{1} \mathbf{K}_{x}^{* \frac{1}{2}}+\mathbf{I} \mid\right) \\
& \simeq \log \left(\left|\mathbf{K}_{x}^{* \frac{1}{2}} \mathbf{G}_{1}^{H} \mathbf{G}_{1} \mathbf{K}_{x}^{* \frac{1}{2}}+2 \mathbf{I}\right|\right. \\
& \left.-\left|\mathbf{K}_{x}^{* \frac{1}{2}} \mathbf{G}_{1}^{H} \mathbf{H}_{2}\left(\mathbf{H}_{2}^{H} \mathbf{H}_{2}\right)^{-1} \mathbf{H}_{2}^{H} \mathbf{G}_{1} \mathbf{K}_{x}^{* \frac{1}{2}}+\mathbf{I}\right|\right) \\
& =\log \left(\left|\mathbf{K}_{x}^{*} \mathbf{G}_{1}^{H} \mathbf{G}_{1}+2 \mathbf{I}\right|\right. \\
& \text { - } \left.\left|\mathbf{H}_{2}\left(\mathbf{H}_{2}^{H} \mathbf{H}_{2}\right)^{-1} \mathbf{H}_{2}^{H} \mathbf{G}_{1} \mathbf{K}_{x}^{*} \mathbf{G}_{1}^{H}+\mathbf{I}\right|\right) \text {. }
\end{aligned}
$$


Equation (30) is obtained from the matrix inversion lemma [20] $(\mathbf{A}+\mathbf{E B ~ F})^{-1}=\mathbf{A}^{-1}-\mathbf{A}^{-1} \mathbf{E}\left(\mathbf{B}^{-1}+\mathbf{F A}^{-1} \mathbf{E}\right)^{-1} \mathbf{F A}{ }^{-1}$.

To show (31), we define

$$
\begin{aligned}
& \mathbf{A}=\mathbf{K}_{x}^{* \frac{1}{2}} \mathbf{G}_{1}^{H} \mathbf{G}_{1} \mathbf{K}_{x}^{* \frac{1}{2}}+2 \mathbf{I} \\
& \mathbf{B}=\mathbf{K}_{x}^{* \frac{1}{2}} \mathbf{G}_{1}^{H} \mathbf{H}_{2} \mathbf{K}_{w}^{\frac{1}{2}} \\
& \times\left(\mathbf{K}_{w}{ }^{\frac{1}{2}} \mathbf{H}_{2}^{H} \mathbf{H}_{2} \mathbf{K}_{w}^{\frac{1}{2}}+\mathbf{I}\right)^{-1} \mathbf{K}_{w}^{\frac{1}{2}} \mathbf{H}_{2}^{H} \mathbf{G}_{1} \mathbf{K}_{x}^{* \frac{1}{2}}+\mathbf{I}
\end{aligned}
$$

and note that $\mathbf{A}-\mathbf{B} \succeq \mathbf{0}$. Moreover, from [20], for any two positive semidefinite matrices $\mathbf{E}$ and $\mathbf{F}$ with the same size, we have

$$
|\mathbf{E}+\mathbf{F}| \geq|\mathbf{E}|+|\mathbf{F}|
$$

Thus $|\mathbf{A}|=|\mathbf{A}-\mathbf{B}+\mathbf{B}| \geq|\mathbf{A}-\mathbf{B}|+|\mathbf{B}|$ and hence $|\mathbf{A}-\mathbf{B}| \leq|\mathbf{A}|-|\mathbf{B}|$, which proves (31). Finally in (32), we assumed that $\left(\mathbf{H}_{2} \mathbf{H}_{2}^{H}\right)^{-1}$ exists $\left(n_{e} \geq n_{h}\right)$, and that the eigenvalues of $\mathbf{K}_{w}{ }^{\frac{1}{2}} \mathbf{H}_{2}^{H} \mathbf{H}_{2} \mathbf{K}_{w}{ }^{\frac{1}{2}}$ are sufficiently bigger than 1, which will hold when $P_{h}$ is large enough. Using (33) in (29), we have the following approximate lower bound on the secrecy rate improvement.

$$
\begin{aligned}
R_{s H}-C_{s} \geq & \log \left|\mathbf{K}_{x}^{*} \mathbf{G}_{1}^{H} \mathbf{G}_{1}+\mathbf{I}\right|-\log \left(\left|\mathbf{K}_{x}^{*} \mathbf{G}_{1}^{H} \mathbf{G}_{1}+2 \mathbf{I}\right|\right. \\
& \left.-\left|\mathbf{H}_{2}\left(\mathbf{H}_{2}^{H} \mathbf{H}_{2}\right)^{-1} \mathbf{H}_{2}^{H} \mathbf{G}_{1} \mathbf{K}_{x}^{*} \mathbf{G}_{1}^{H}+\mathbf{I}\right|\right) .
\end{aligned}
$$

In Section V, we will provide a numerical example to compare the bound in (35) with the actual improvement obtained by our proposed approach.

\section{RESUlTS FOR DIFFERENT SCENARIOS}

Thus far, we have not made any assumption on the number of antennas at each node. It is clear from (20) that, for example when $\mathbf{G}_{2}$ has more columns than rows, for a fixed $\mathbf{N}$ in the acceptable range (21) there will be an infinite number of $\mathbf{K}_{w}$ matrices that satisfy (20) and consequently do not decrease $I\left(X_{1} ; Y_{1}\right)$. In fact, in this example, a common policy for the helper is to simply transmit artificial noise in the null space of $\mathbf{G}_{2}$. A more interesting case occurs when no such null space exists, i.e., when the number of antennas at the helper is less than or equal to that of the intended receiver $\left(n_{h} \leq n_{r}\right)$. The previous section demonstrates the nontrivial fact that even when $n_{h} \leq n_{r}$, it is possible to find a nonzero jamming signal that does not impact $I\left(X_{1} ; Y_{1}\right)$ even when the jamming signal can not be nulled by the channel. In the discussion below, we find more constructive expressions for the $\mathbf{K}_{w}$ matrices that satisfy (20) for various combinations of the number of antennas at different nodes. We will consider all possible combinations of the number of antennas at the transmitter, helper and intended receiver. In particular, we will show that when $n_{h} \leq n_{r}$, a closed-form expression for $\mathbf{K}_{w}$ can be found.

Such $\mathbf{K}_{w}$ will have no impact on $I\left(X_{1} ; Y_{1}\right)$, but will in general decrease $I\left(X_{1} ; Y_{2}\right)$, compared with the case that there is no helper. Hence, the secrecy level of the confidential message is increased. As mentioned earlier, additional improvement in the secrecy rate beyond that achievable by using a nonzero $\mathbf{K}_{w}$ can be achieved if the transmitter updates its covariance matrix $\mathbf{K}_{x}$ once $\mathbf{K}_{w}$ is computed. Note, however, that such an iterative process will not be pursued beyond updating $\mathbf{K}_{x}$; unlike the first step, where $\mathbf{K}_{w}$ was updated from its initial value of zero, there is no guarantee that finding a new $\mathbf{K}_{w}$ in a second step will reduce $I\left(X_{1} ; Y_{2}\right)$. Hence, the final secrecy rate for the proposed method is obtained by simply computing (6) and (7) for the resulting $\mathbf{K}_{w}$ matrices derived in this section.

\section{A. Case 1: $n_{h} \leq \min \left\{n_{r}, n_{t}\right\}$}

We show here that for the case where $n_{h} \leq \min \left\{n_{r}, n_{t}\right\}$ and for a fixed $\mathbf{N}$ in the acceptable range (21), there is only one $\mathbf{K}_{w}$ matrix that satisfies (20) and consequently does not decrease $I\left(X_{1} ; Y_{1}\right)$. Using the matrix inversion lemma, (20) can be written as

$$
\begin{aligned}
\mathbf{H}_{1}^{H} & \left(\mathbf{G}_{2} \mathbf{K}_{w} \mathbf{G}_{2}^{H}+\mathbf{I}\right)^{-1} \mathbf{H}_{1} \\
= & \mathbf{H}_{1}^{H} \mathbf{H}_{1}-\mathbf{H}_{1}^{H} \mathbf{G}_{2}\left(\mathbf{G}_{2}^{H} \mathbf{G}_{2}+\mathbf{K}_{w}^{-1}\right)^{-1} \mathbf{G}_{2}^{H} \mathbf{H}_{1} \\
= & \mathbf{S}^{-1 / 2}\left[\mathbf{C}^{-H}\left[\begin{array}{cc}
\mathbf{\Lambda}_{1} & 0 \\
0 & \mathbf{N}
\end{array}\right] \mathbf{C}^{-1}-\mathbf{I}\right] \mathbf{S}^{-1 / 2} .
\end{aligned}
$$

Replacing $\mathbf{H}_{1}^{H} \mathbf{H}_{1}$ with (14), we have:

$$
\begin{aligned}
\mathbf{H}_{1}^{H} \mathbf{G}_{2}\left(\mathbf{G}_{2}^{H} \mathbf{G}_{2}+\mathbf{K}_{w}^{-1}\right)^{-1} \mathbf{G}_{2}^{H} \mathbf{H}_{1} \\
=\mathbf{S}^{-1 / 2} \mathbf{C}^{-H}\left[\begin{array}{cc}
0 & 0 \\
0 & \boldsymbol{\Lambda}_{2}-\mathbf{N}
\end{array}\right] \mathbf{C}^{-1} \mathbf{S}^{-1 / 2} .
\end{aligned}
$$

Since we have assumed that the channels are full rank, for the case of $n_{h} \leq n_{r} \leq n_{t}$ or $n_{h} \leq n_{t} \leq n_{r}$, it is clear that rank $\left(\mathbf{G}_{2}^{H} \mathbf{H}_{1}\right)=n_{h}$. Thus, from (36) we have

$$
\begin{aligned}
\left(\mathbf{G}_{2}^{H} \mathbf{G}_{2}+\mathbf{K}_{w}^{-1}\right)^{-1}= & \mathbf{O}^{H} \mathbf{S}^{-1 / 2} \mathbf{C}^{-H} \\
& \times\left[\begin{array}{cc}
0 & 0 \\
0 & \boldsymbol{\Lambda}_{2}-\mathbf{N}
\end{array}\right] \mathbf{C}^{-1} \mathbf{S}^{-1 / 2} \mathbf{O}
\end{aligned}
$$

where $\mathbf{O}$ is the right inverse of $\mathbf{G}_{2}^{H} \mathbf{H}_{1}$, which, for example when $n_{h} \leq n_{r} \leq n_{t}$, can be written as $\mathbf{O}=\mathbf{H}_{1}^{H}\left(\mathbf{H}_{1} \mathbf{H}_{1}^{H}\right)^{-1} \overline{\mathbf{G}}_{2}\left(\mathbf{G}_{2}^{H} \mathbf{G}_{2}\right)^{-1}$. The following lemma is a direct result of (36) and (37).

Lemma 3: For the case of $n_{h} \leq \min \left\{n_{r}, n_{t}\right\}$ and for a fixed $\mathbf{N}$ in the acceptable range (21), the $\mathbf{K}_{w} \succeq 0$ matrix for which (20) is satisfied and $I\left(X_{1} ; Y_{1}\right)$ is not decreased is given by

$$
\mathbf{K}_{w}=\mathbf{Q}-\mathbf{Q G}_{2}^{H}\left(\mathbf{G}_{2} \mathbf{Q G}_{2}^{H}-\mathbf{I}\right)^{-1} \mathbf{G}_{2} \mathbf{Q}
$$

where $\mathbf{Q}$ is the RHS of (37).

Proof: After applying the matrix inversion lemma on the LHS of (37), a straightforward computation yields (38).

As is evident from (37)-(38), we still have a design parameter, $\mathbf{N}$, that should be chosen in its acceptable range $\boldsymbol{\Lambda}_{22} \preceq$ $\mathbf{N} \preceq \Lambda_{2}$ such that the power constraint $\operatorname{Tr}\left(\mathbf{K}_{w}\right)=P_{h}$ is satisfied. Finding the optimal $\mathbf{N}$ that minimizes $I\left(X_{1} ; Y_{2}\right)$ when $\mathbf{K}_{x}$ and $\mathbf{K}_{w}$ are given by (13) and (38), respectively, is as intractable as the general optimization problem in (5). Instead, we simply restrict the $\mathbf{N}$ we consider to those that can be linearly parameterized within the acceptable range, as follows:

$$
\mathbf{N}=\boldsymbol{\Lambda}_{22}+t\left(\boldsymbol{\Lambda}_{2}-\boldsymbol{\Lambda}_{22}\right) \text {. }
$$

Consequently the term $\boldsymbol{\Lambda}_{2}-\mathbf{N}$ in (38) becomes

$$
\boldsymbol{\Lambda}_{2}-\mathbf{N}=(1-t)\left(\boldsymbol{\Lambda}_{2}-\boldsymbol{\Lambda}_{22}\right)
$$


where the scalar $0 \leq t \leq 1$ is chosen such that the power constraint $\operatorname{Tr}\left(\mathbf{K}_{w}\right)=P_{h}$ is satisfied. Note that as $t \rightarrow 0(\mathbf{N} \rightarrow$ $\left.\boldsymbol{\Lambda}_{22}\right)$ then $\operatorname{Tr}\left(\mathbf{K}_{w}\right) \rightarrow \infty$, and as $t \rightarrow 1\left(\mathbf{N} \rightarrow \boldsymbol{\Lambda}_{2}\right)$ then $\operatorname{Tr}\left(\mathbf{K}_{w}\right) \rightarrow 0$. Thus, we are guaranteed that an acceptable $\mathbf{N}$ can be found in this way.

\section{B. Case 2: $n_{h}>\min \left\{n_{r}, n_{t}\right\}$}

As mentioned before, for the case of $n_{h}>n_{r}$ and for a fixed $\mathbf{N}$ in the acceptable range (21), there are many $\mathbf{K}_{w}$ matrices that satisfy (20) and consequently do not decrease $I\left(X_{1} ; Y_{1}\right)$. A common policy for the helper in this case is to transmit artificial noise in the null space of $\mathbf{G}_{2}$. However, as (20) shows, this policy is sufficient but it is not necessary. In other words, it is possible that the optimal $\mathbf{K}_{w}$ satisfying (20) has elements outside the null space of $\mathbf{G}_{2}$. Because of the nonlinear constraint in (20), finding the optimal $\mathbf{K}_{w}$ is intractable. A similar discussion applies for the case of $n_{t}<n_{h} \leq n_{r}$.

In this section, we present an approach for computing a suitable $\mathbf{K}_{w}$. Consider the following jamming signal covariance matrix:

$$
\mathbf{K}_{w}=\Gamma \Pi \Gamma^{H},
$$

where $\Pi$ is a $d \times d$ positive semidefinite matrix, and $\boldsymbol{\Gamma}$ is an $n_{h} \times d$ matrix. For the case of $n_{t}<n_{h} \leq n_{r}$ or $n_{h}>n_{r}$, we can choose $\boldsymbol{\Gamma}$ such that $\mathbf{G}_{2} \boldsymbol{\Gamma}$ is orthogonal to $\mathbf{H}_{1} \mathbf{K}_{x}^{* \frac{1}{2}}$, i.e., $\mathbf{K}_{x}^{* \frac{1}{2}} \mathbf{H}_{1}^{H} \mathbf{G}_{2} \boldsymbol{\Gamma}=\mathbf{0}$. For example, $\boldsymbol{\Gamma}$ can be chosen as the $d$ right singular vectors in the nullspace of $\mathbf{K}_{x}^{* \frac{1}{2}} \mathbf{H}_{1}^{H} \mathbf{G}_{2}$. Since $\mathbf{K}_{x}$ will often be rank deficient, the value of $d$ will typically be larger than $n_{h}-n_{t}$ for the case of $n_{t}<n_{h} \leq n_{r}$, and larger than $n_{h}-n_{r}$ for the case of $n_{h}>n_{r}$. For this choice of $\boldsymbol{\Gamma}$, the resulting $\mathbf{K}_{w}$ in (40) satisfies (20), and doesn't decrease $I\left(X_{1} ; Y_{1}\right)$ for $\mathbf{N}=\boldsymbol{\Lambda}_{2}$, as is clear from (20). Given $\boldsymbol{\Gamma}$, the choice of $\boldsymbol{\Pi}$ can be made to maximize the transfer of the "information" in the helper's jamming signal to the eavesdropper. In particular, note that at the eavesdropper, the covariance of the helper's jamming signal will be given by $\mathbf{H}_{2} \boldsymbol{\Gamma} \boldsymbol{\Pi} \boldsymbol{\Gamma}^{H} \mathbf{H}_{2}^{H}$. If the eigenvalue decomposition of $\boldsymbol{\Gamma}^{H} \mathbf{H}_{2}^{H} \mathbf{H}_{2} \boldsymbol{\Gamma}$ is written as

$$
\boldsymbol{\Gamma}^{H} \mathbf{H}_{2}^{H} \mathbf{H}_{2} \boldsymbol{\Gamma}=\mathbf{U} \mathbf{D} \mathbf{U}^{H}
$$

with $\mathbf{U}$ unitary and $\mathbf{D}$ square and diagonal, then $\boldsymbol{\Pi}$ can be found via waterfilling; i.e.

$$
\Pi=\mathbf{U} \boldsymbol{\Delta} \mathbf{U}^{H},
$$

where $\boldsymbol{\Delta}=\left[\eta \mathbf{I}-\mathbf{D}^{-1}\right]^{+}$, the operation $[\mathbf{A}]^{+}$zeros out any negative elements, and the water-filling level $\eta$ is chosen such that $\operatorname{Tr}\left(\mathbf{K}_{w}\right)=\operatorname{Tr}(\boldsymbol{\Delta})=P_{h}$.

\section{NUMERICAL RESULTS}

In this section, we present numerical results to illustrate our theoretical findings. In all of the following figures, channels are assumed to be quasi-static flat Rayleigh fading and independent of each other. The channel matrices $\mathbf{H}_{1} \in \mathbb{C}^{n_{r} \times n_{t}}$ and $\mathbf{G}_{2} \in \mathbb{C}^{n_{r} \times n_{h}}$ have i.i.d. entries distributed as $\mathcal{C N}\left(0, \sigma_{d}^{2}\right)$, while $\mathbf{G}_{1} \in \mathbb{C}^{n_{e} \times n_{t}}$ and $\mathbf{H}_{2} \in \mathbb{C}^{n_{e} \times n_{h}}$ have i.i.d. entries distributed as $\mathcal{C N}\left(0, \sigma_{c}^{2}\right)$. In each figure, values for the number of antennas at each node, as well as $\sigma_{d}^{2}$ and $\sigma_{c}^{2}$, will be depicted.

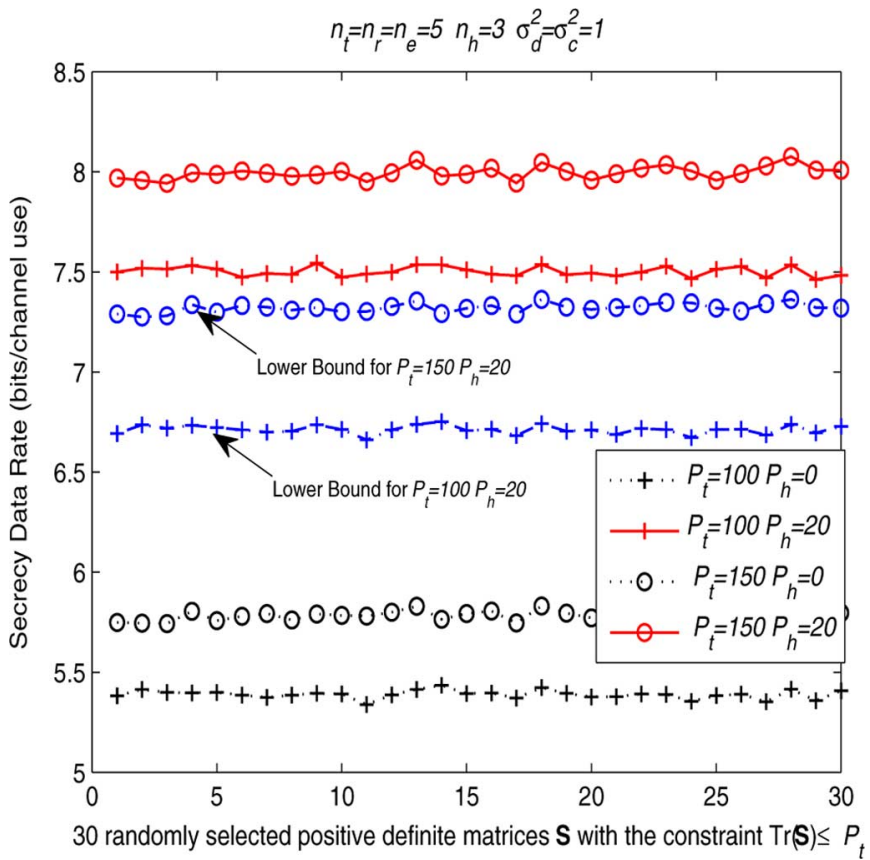

Fig. 2. Comparison of the achievable secrecy data rate for MIMO Gaussian wiretap channel with and without helper for different $P_{t}$ and $P_{h}$.

Unless otherwise indicated, results are calculated based on an average of at least 500 independent channel realizations.

In the first example, Fig. 2, we randomly generate positive definite matrices $\mathbf{S}$ such that $\operatorname{Tr}(\mathbf{S}) \leq P_{t}$. For each $\mathbf{S}$, we compute the secrecy capacity of the MIMO Gaussian wiretap channel without helper $\left(\mathbf{K}_{w}=\mathbf{0}\right)$ as given by (12). Next, using (38), we obtain a $\mathbf{K}_{w}$ with the average power constraint $\operatorname{Tr}\left(\mathbf{K}_{w}\right)=P_{h}$ that does not decrease $I\left(X_{1} ; Y_{1}\right)$, and then update $\mathbf{K}_{x}$ and compute $R_{\text {sec }}(\mathbf{S})$, using (6) and (7), accordingly. For each $\mathbf{S}$, we also calcluate the approximate lower bound on the secrecy rate improvement offered by the proposed algorithm, as given by (35). Fig. 2 compares the secrecy rate of the wiretap channel with (solid lines) and without (dotted lines) the helper, together with the bound (dash-dotted lines). Note that the vertical difference between the solid curves (about 0.5 bits/channel use) represents the role of the transmit power $P_{t}$ on the secrecy rate with helper when $P_{t}$ changes from 100 to 150 and $P_{h}=20$. This relatively small difference indicates that, in this example, $P_{t}$ does not have a big impact on the secrecy rate. Its role is even more negligible when $P_{h}=0$, where only an increase of $0.3 \mathrm{bits} / \mathrm{channel}$ use is obtained as $P_{t}$ increases from 100 to 150 . The role of the helper on the other hand is significantly more important; increasing $P_{h}$ from 0 to 20 while holding $P_{t}$ fixed results in an increase on the order of $2.3 \mathrm{bits} / \mathrm{channel}$ use. Furthermore, the use of the helper with a total power of only $120\left(P_{t}=100, P_{h}=20\right)$ provides significantly better secrecy performance than not using the helper and transmitting with total power equal to $150\left(P_{t}=150, P_{h}=0\right)$. Comparing the gap between the curves for a given transmit power $P_{t}$, we observe that for both scenarios, the gap between the lower bound and the achievable secrecy rate is much larger for the case with no helper $\left(P_{h}=0\right)$ than when a helper is present $\left(P_{h}=20\right)$.

In the next examples, we calculate the maximum achievable secrecy rate of the proposed algorithms under the assumption 


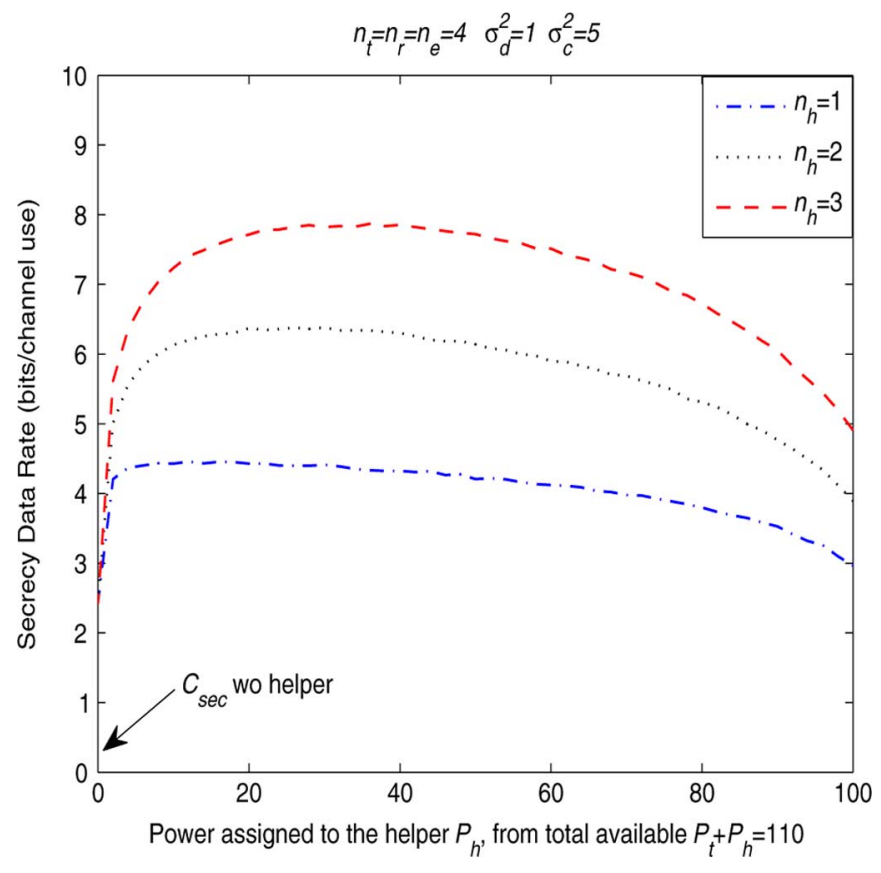

Fig. 3. Comparison of the achievable secrecy data rate for the MIMO Gaussian wiretap channel with and without a helper versus $P_{h}$ for different number of antennas at the helper, $P_{t}+P_{h}=110$, assuming the eavesdropper's channels are stronger than those of the receiver $\left(\sigma_{d}^{2}=1, \sigma_{c}^{2}=5\right)$.

of an average power constraint $P_{t}$ at the transmitter, and under the constraint that the helper does not reduce the mutual information between the transmitter and receiver. While (6) and (7) provide the performance for a specific $\mathbf{S}$, one must solve [19], [22, Lemma 1]

$$
R_{\mathrm{sec}}\left(P_{t}\right)=\max _{\mathbf{S} \succeq 0, \operatorname{Tr}(\mathbf{S}) \leq P_{t}} R_{\mathrm{sec}}(\mathbf{S})
$$

to find the maximum achievable secrecy rate over all $\mathbf{S}$ that satisfy the average power constraint. In the examples that follow, we perform a numerical search to solve (41) and compute $R_{\mathrm{sec}}\left(P_{t}\right)$.

Fig. 3 shows the maximum achievable secrecy rate for the proposed algorithm versus $P_{h}$ for a fixed total average power $P_{t}+P_{h}=110$. In this figure, we consider a situation in which $\sigma_{c}>\sigma_{d}$, or in other words where the channel between the transmitter and the intended receiver is weaker than the channel between the transmitter and the eavesdropper, and the channel between the helper and the intended receiver is weaker than the channel between the helper and the eavesdropper. The arrow in the figure shows the secrecy capacity without the helper $\left(P_{h}=\right.$ $0)$. The figure shows that a helper with just a single antenna can provide a dramatic improvement in secrecy rate with very little power allocated to the jamming signal; in fact, the optimal rate is obtained when $P_{h}$ is less than $2 \%$ of the total available transmit power. If the number of antennas at the helper increases, a much higher secrecy rate can be obtained, but at the expense of allocating more power to the helper and less to the signal for the desired user.

In Fig. 4, we consider a situation in which, unlike the above example, we have $\sigma_{d}>\sigma_{c}$. Thus, the intended receiver, in comparison with the eavesdropper, receives a weaker information signal and a stronger jamming signal than the eavesdropper.

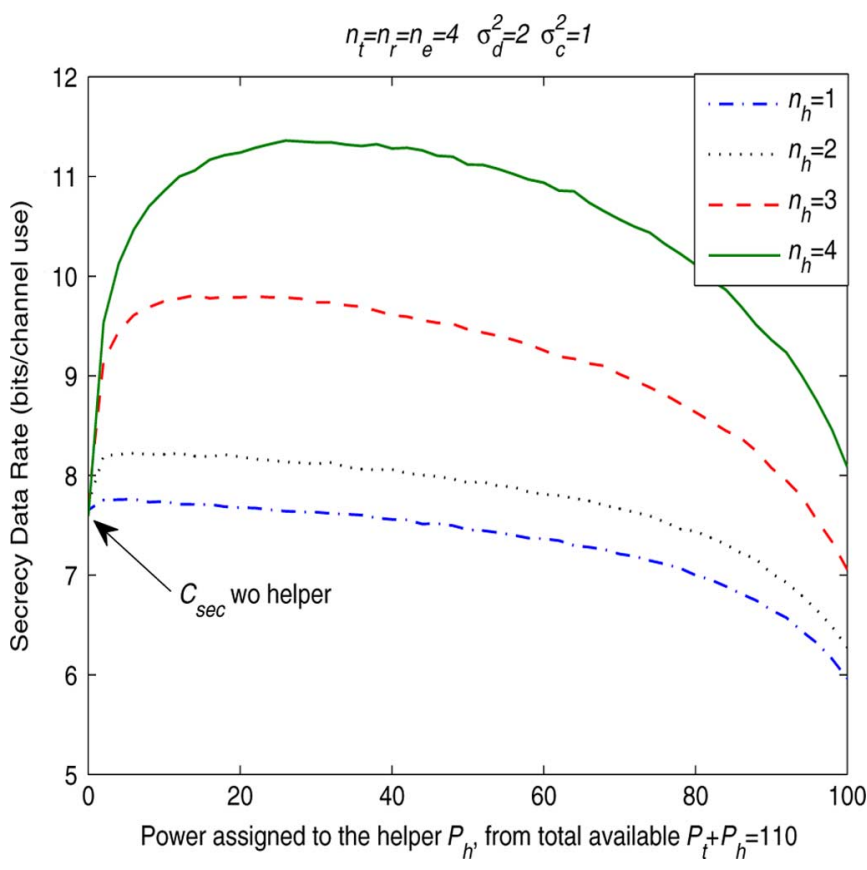

Fig. 4. Comparison of the achievable secrecy data rate for the MIMO Gaussian wiretap channel with and without a helper versus $P_{h}$ for different number of antennas at the helper, $P_{t}+P_{h}=110$, assuming the receiver's channels are stronger than those of the eavesdropper $\left(\sigma_{d}^{2}=2, \sigma_{c}^{2}=1\right)$.

It might seem that in this situation, the helper cannot be very useful, but the figure shows that even in this case we can have a notable improvement in the secrecy rate (about 4 bits/channel use) by increasing the number of antennas at the helper, and with an appropriate power assignment between the transmitter and the helper, without requiring extra total transmit power for the helper node.

In Fig. 5, we consider a specific scenario where the secrecy capacity in the absence of the helper node is zero. While channel matrices $\mathbf{H}_{2}$ and $\mathbf{G}_{2}$ are generated randomly with i.i.d. entries distributed as $\mathcal{C N}\left(0, \sigma_{c}^{2}\right)$ and $\mathcal{C N}\left(0, \sigma_{d}^{2}\right)$, respectively, we assume the following specific choices for $\mathbf{H}_{1}$ and $\mathbf{G}_{1}$ :

$$
\begin{aligned}
\mathbf{H}_{1} & =\left[\begin{array}{ccc}
-0.25+0.5 i & -0.35 & -1.25-0.9 i \\
-0.4+0.1 i & -0.2+0.75 i & -i
\end{array}\right] \\
\mathbf{G}_{1} & =\left[\begin{array}{ccc}
2+0.25 i & 1.5+0.5 i & 2 i \\
0.25+0.25 i & -0.7+1.5 i & 0.5+0.33 i \\
-1.5 & -0.5-i & -2.9 i
\end{array}\right] .
\end{aligned}
$$

Since $\mathbf{H}_{1}^{H} \mathbf{H}_{1} \preceq \mathbf{G}_{1}^{H} \mathbf{G}_{1}$, all the generalized eigenvalues of the pencil

$$
\left(\mathbf{S}^{\frac{1}{2}} \mathbf{H}_{1}^{H} \mathbf{H}_{1} \mathbf{S}^{\frac{1}{2}}+\mathbf{I}, \mathbf{S}^{\frac{1}{2}} \mathbf{G}_{1}^{H} \mathbf{G}_{1} \mathbf{S}^{\frac{1}{2}}+\mathbf{I}\right)
$$

are zero for all $\mathbf{S} \succeq 0$, and consequently the secrecy capacity without helper will be zero. In this example, we also assume that not only is the total power fixed at $P_{t}+P_{h}=110$, but also the total number of transmit antennas is fixed at $n_{t}+n_{h}=3$. As in the other examples, the secrecy rate of the wiretap channel is considerably improved with the helper. In this case, the best performance is obtained when the helper has only a single antenna.

Finally, in Fig. 6, we consider the role of number of antennas at the helper, $n_{h}$, in the secrecy rate for the specific matrix power 


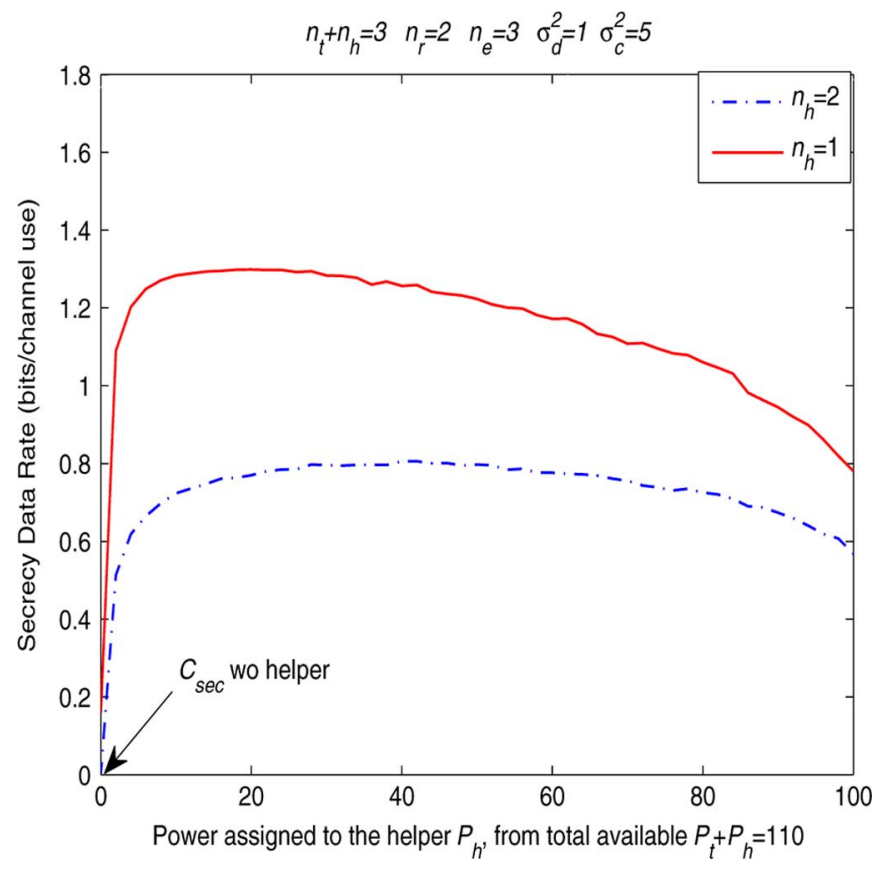

Fig. 5. Comparison of the achievable secrecy data rate for the MIMO Gaussian wiretap channel with and without a helper versus $P_{h}$ for different number of antennas at the helper, $P_{t}+P_{h}=110$, and $n_{t}+n_{h}=3$.

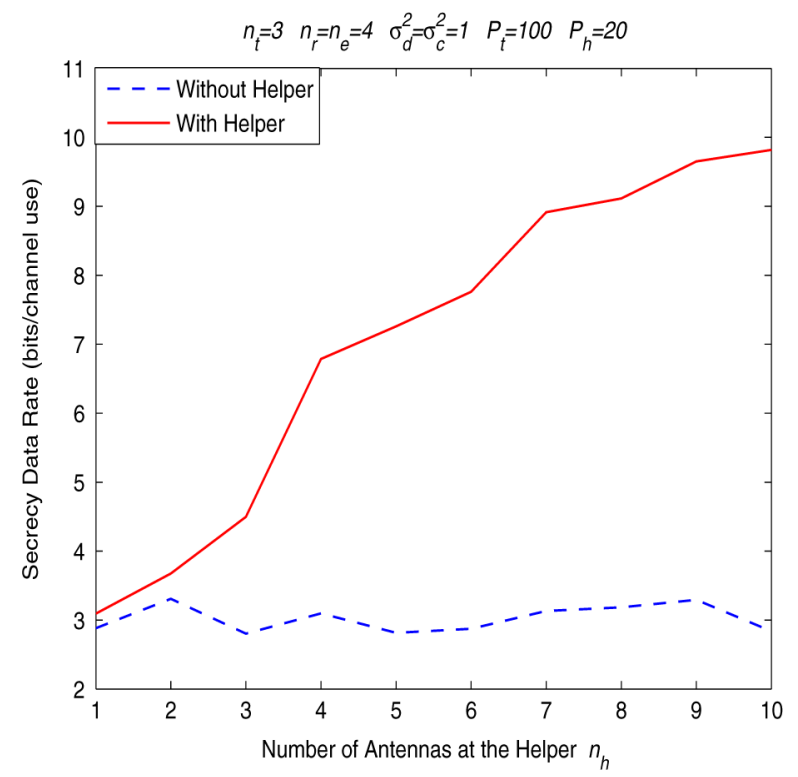

Fig. 6. Secrecy data rate versus $n_{h}$ for a specific matrix power constraint $\mathbf{S}=$ $\frac{P_{t}}{n_{t}} \mathbf{I}$.

constraint $\mathbf{S}=\frac{P_{t}}{n} \mathbf{I}$. Note that the solution of Section IV-A applies for $n_{h} \leq 3$, while the solution of Section IV-B holds for $n_{h}>3$. In all cases, we see that the secrecy rate increases considerably as $n_{h}$ increases.

\section{CONCLUSION}

In this paper, we have studied the Gaussian MIMO Wiretap channel in the presence of an external jammer/helper, where the helper node assists the transmitter by sending artificial noise independent of the source message to confuse the eavesdropper.
The jamming signal from the helper is not required to be decoded by the intended receiver and is treated as noise at both the intended receiver and the eavesdropper. We obtained a closedform relationship for the structure of the helper's artificial noise covariance matrix that guarantees no decrease in the mutual information between the transmitter and the intended receiver. We also derived an approximate lower bound on the improvement in secrecy rate obtained when jamming signals that satisfy this constraint are employed. We showed how to construct specific solutions within this covariance matrix framework that provide very good secrecy rate performance, even when there is no nontrivial null space between the helper and the intended receiver. Our numerical results demonstrated that the proposed scheme achieves a notable improvement in secrecy rate even for a fixed average total power and a fixed total number of antennas at the transmitter and the helper, without requiring extra power or antennas to be allocated to the helper node.

\section{APPENDIX A}

We are interested in finding a relationship that represents all matrices $\Sigma \succ 0$ for which

$$
\log \left|\mathbf{K}_{x}^{*} \boldsymbol{\Sigma}+\mathbf{I}\right|=\log \left|\left(\mathbf{C}_{1}^{H} \mathbf{C}_{1}\right)^{-1} \mathbf{\Lambda}_{1}\right|
$$

where

$$
\mathbf{K}_{x}^{*}=\mathbf{S}^{\frac{1}{2}} \mathbf{C}\left[\begin{array}{cc}
\left(\mathbf{C}_{1}^{H} \mathbf{C}_{1}\right)^{-1} & 0 \\
0 & 0
\end{array}\right] \mathbf{C}^{H} \mathbf{S}^{\frac{1}{2}}
$$

Using the fact that $|\mathbf{A B}+\mathbf{I}|=|\mathbf{B A}+\mathbf{I}|$, it is clear that $\mathbf{\Sigma}$ will have the form $\boldsymbol{\Sigma}=\mathbf{S}^{-\frac{1}{2}} \mathbf{C}^{-H} \mathbf{X} \mathbf{C}^{-1} \mathbf{S}^{-\frac{1}{2}}$ for some matrix $\mathbf{X}=\mathbf{X}^{H}$. Substituting this expression for $\boldsymbol{\Sigma}$ into (42) results in the following equation that must be solved for $\mathbf{X}$ :

$$
\log \left|\left[\begin{array}{cc}
\left(\mathbf{C}_{1}^{H} \mathbf{C}_{1}\right)^{-1} & 0 \\
0 & 0
\end{array}\right] \mathbf{X}+\mathbf{I}\right|=\log \left|\left(\mathbf{C}_{1}^{H} \mathbf{C}_{1}\right)^{-1} \mathbf{\Lambda}_{1}\right|
$$

Write $\mathbf{X}$ as $\mathbf{X}=\left[\begin{array}{ll}\mathbf{X}_{1} & \mathbf{X}_{2} \\ \mathbf{X}_{2}^{H} & \mathbf{X}_{3}\end{array}\right]$ so that we have

$$
\begin{aligned}
{\left[\begin{array}{cc}
\left(\mathbf{C}_{1}^{H} \mathbf{C}_{1}\right)^{-1} & 0 \\
0 & 0
\end{array}\right] \mathbf{X}+\mathbf{I} } & \\
& =\left[\begin{array}{cc}
\left(\mathbf{C}_{1}^{H} \mathbf{C}_{1}\right)^{-1} \mathbf{X}_{1}+\mathbf{I} & \left(\mathbf{C}_{1}^{H} \mathbf{C}_{1}\right)^{-1} \mathbf{X}_{2} \\
0 & \mathbf{I}
\end{array}\right]
\end{aligned}
$$

and note that the determinant of the above matrix is given by $\left|\left(\mathbf{C}_{1}^{H} \mathbf{C}_{1}\right)^{-1} \mathbf{X}_{1}+\mathbf{I}\right|$. By comparing this result to (42), we see that $\mathbf{X}_{1}=\mathbf{\Lambda}_{1}-\left(\mathbf{C}_{1}^{H} \mathbf{C}_{1}\right)$. Consequently, we have

$$
\boldsymbol{\Sigma}=\mathbf{S}^{-\frac{1}{2}} \mathbf{C}^{-H}\left[\begin{array}{cc}
\boldsymbol{\Lambda}_{1}-\underset{\mathbf{X}_{2}^{H}}{\left(\mathbf{C}_{1}^{H} \mathbf{C}_{1}\right)} & \mathbf{X}_{2} \\
\mathbf{X}_{3}
\end{array}\right] \mathbf{C}^{-1} \mathbf{S}^{-\frac{1}{2}}
$$

where $\mathbf{X}_{2}$ and $\mathbf{X}_{3}$ are still unknown and must be found as described in the text. It is clear that (45) and (22) are equivalent.

\section{REFERENCES}

[1] A. Wyner, "The wire-tap channel," Bell Syst. Tech. J., vol. 54, no. 8, pp. 1355-1387, Jan. 1975.

[2] S. K. Leung-Yan-Cheong and M. E. Hellman, "The Gaussian wire-tap channel," IEEE Trans. Inf. Theory, vol. 24, pp. 451-456, Jul. 1978. 
[3] F. Oggier and B. Hassibi, "The secrecy capacity of the MIMO wiretap channel," in Proc. IEEE Int. Symp. Inf. Theory, Toronto, ON, Canada, Jul. 2008, pp. 524-528.

[4] A. Khisti and G. Wornell, "Secure transmission with multiple antennas II: The MIMOME wiretap channel," IEEE Trans. Inf. Theory, vol. 56, no. 11 , pp. 5515-5532, 2010.

[5] J. Li and A. Petropulu, "Transmitter optimization for achieving secrecy capacity in Gaussian MIMO wiretap channels," IEEE Trans. Inf. Theory [Online]. Available: http://arxiv.org/abs/0909.2622, submitted for publication

[6] T. Liu and S. Shamai (Shitz), "A note on secrecy capacity of the multiantenna wiretap channel," IEEE Trans. Inf. Theory, vol. 55, no. 6, pp. 2547-2553, 2009.

[7] R. Bustin, R. Liu, H. V. Poor, and S. Shamai (Shitz), "A MMSE approach to the secrecy capacity of the MIMO Gaussian wiretap channel," EURASIP J. Wireless Commun. Netw., vol. 2009, p. 8, 2009, Article ID 370970.

[8] R. Liu, T. Liu, H. V. Poor, and S. Shamai (Shitz), "New results on multiple-input multiple-output broadcast channels with confidential messages," IEEE Trans. Inf. Theory [Online]. Available: http://arxiv.org/PS-cache/arxiv/pdf/1101/1101.2007v1.pdf, submitted for publication

[9] G. Bagherikaram, A. S. Motahari, and A. K. Khandani, "The secrecy capacity region of the Gaussian MIMO broadcast channel," IEEE Trans. Inf. Theory Mar. 2009 [Online]. Available: http://arxiv.org/ PScache/arxiv/pdf/0903/0903.3261v2.pdf, submitted for publication

[10] I. Csiszàr and J. Körner, "Broadcast channels with confidential messages," IEEE Trans. Inf. Theory, vol. 24, no. 3, pp. 339-348, May 1978.

[11] E. Tekin and A. Yener, "The general Gaussian multiple access and two-way wire-tap channels: Achievable rates and cooperative jamming," IEEE Trans. Inf. Theory, vol. 54, no. 6, pp. 2735-2751, Jun. 2008.

[12] L. Dong, Z. Han, A. P. Petropulu, and H. V. Poor, "Cooperative jamming for wireless physical layer security," in Proc. IEEE Workshop on Statistical Signal Process., Cardiff, Wales, U.K., 2009.

[13] L. Dong, Z. Han, A. P. Petropulu, and H. V. Poor, "Improving wireless physical layer security via cooperating relays," IEEE Trans. Signal Process., vol. 58, no. 3, pp. 1875-1888, Mar. 2010.

[14] L. Lai and H. El Gamal, "The relay-eavesdropper channel: Cooperation for secrecy," IEEE Trans. Inf. Theory, vol. 54, no. 9, pp. 4005-4019, Sep. 2008.

[15] X. Tang, R. Liu, P. Spasojevic, and H. V. Poor, "The Gaussian wiretap channel with a helping interferer," in Proc. IEEE Int. Symp. Inf. Theory, Toronto, ON, Canada, Jul. 2008.

[16] J. Wang and A. L. Swindlehurst, "Cooperative jamming in MIMO ad hoc networks," in Proc. Asilomar Conf. Signals, Syst. Comput., Nov. 2009, pp. 1719-1723.

[17] E. MolavianJazi, M. Bloch, and J. N. Laneman, "Arbitrary jamming can preclude secure communication," in Proc. Allerton Conf. Commun., Contr., Comput., Monticello, IL, Sep. 2009.

[18] S. Goel and R. Negi, "Guaranteeing secrecy using artificial noise," IEEE Trans. Wireless Commun., vol. 7, no. 6, pp. 2180-2189, Jun. 2008.

[19] R. Liu, T. Liu, H. V. Poor, and S. Shamai (Shitz), "Multiple-input multiple-output Gaussian broadcast channels with confidential messages," IEEE Trans. Inf. Theory, vol. 56, no. 9, pp. 4215-4227, Sept. 2010.

[20] R. A. Horn and C. R. Johnson, Matrix Analysis. Cambridge, U.K.: Cambridge Univ. Press, 1985.
[21] S. W. Peters and R. W. Heath, Jr., "Interference alignment via alternating minimization," in Proc. IEEE ICASSP, Taiwan, Apr. 2009.

[22] H. Weingarten, Y. Steinberg, and S. Shamai (Shitz), "The capacity region of the Gaussian multiple-input multiple-output broadcast channel," IEEE Trans. Inf. Theory, vol. 52, no. 9, pp. 3936-3964, 2006.

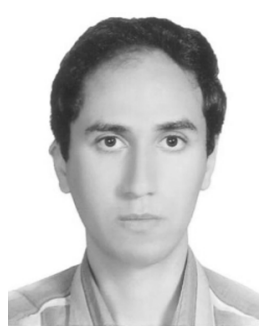

S. Ali. A. Fakoorian (S'11) received the B.S. degree in 2002, and the M.S. degree in 2005, from Amirkabir University of Technology, Tehran, Iran, both in electrical engineering.

$\mathrm{He}$ is currently pursuing the Ph.D. degree at the Department of Electrical Engineering and Computer Science, University of California, Irvine. His research interests lie in multiple-user wireless networks, signal processing and information theory.

Mr. Fakoorian is a student member of CPCC and the IEEE Communications and Signal Processing So-

cieties.

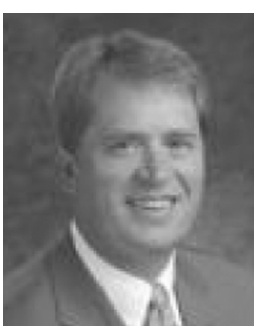

A. Lee Swindlehurst (S'83-M'90-SM'99-F'04) received the B.S. (summa cum laude) and M.S. degrees in electrical engineering from Brigham Young University, Provo, UT, in 1985 and 1986, respectively, and the Ph.D. degree in electrical engineering from Stanford University, Stanford, CA, in 1991.

From 1986 to 1990, he was with ESL, Inc., Sunnyvale, CA, where he was involved in the design of algorithms and architectures for several radar and sonar signal processing systems. He was on the faculty of the Department of Electrical and Computer Engineering, Brigham Young University, from 1990 to 2007, where he was a Full Professor and served as Department Chair from 2003 to 2006. During 1996-1997, he held a joint appointment as a Visiting Scholar at both Uppsala University, Uppsala, Sweden, and at the Royal Institute of Technology, Stockholm, Sweden. From 2006 to 2007, he was on leave working as Vice President of Research for ArrayComm LLC, San Jose, CA. He is currently a Professor of electrical engineering and computer science with the University of California at Irvine. His research interests include sensor array signal processing for radar and wireless communications, detection and estimation theory, and system identification. He has more than 200 publications in these areas.

Dr. Swindlehurst is past Secretary of the IEEE Signal Processing Society, past Editor-in-Chief of the IEEE JOURNAL OF SELECTED TOPICS IN Signal Processing, and past member of the Editorial Boards for the EURASIP Journal on Wireless Communications and Networking, IEEE Signal Processing Magazine, and the IEEE Transactions on Signal PROCESSING. He is a recipient of several paper awards: the 2000 IEEE W. R. G. Baker Prize Paper Award, the 2006 and 2010 IEEE Signal Processing Societys Best Paper Award, the 2006 IEEE Communications Society Stephen O. Rice Prize in the Field of Communication Theory, and is coauthor of a paper that received the IEEE Signal Processing Society Young Author Best Paper Award in 2001. 